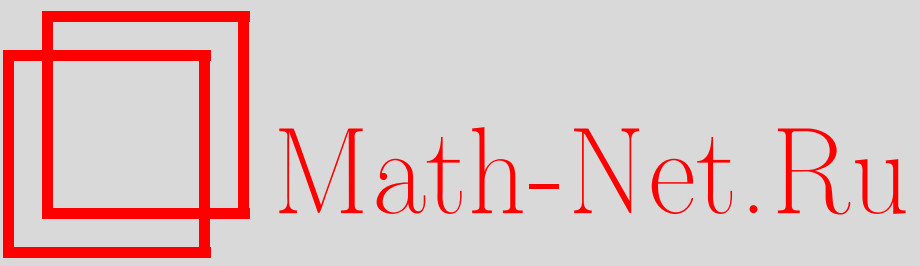

С. В. Людковский, Квазиинвариантные меры на неархимедовых полугруппах петель, УМН, 1998, том 53, выпуск 3, 203-204

DOI: https://doi.org/10.4213/rm51

Использование Общероссийского математического портала Math-Net.Ru подразумевает, что вы прочитали и согласны с пользовательским соглашением

http://www . mathnet.ru/rus/agreement

Параметры загрузки:

IP: 52.90 .164 .192

26 апреля 2023 г., 17:31:16 


\section{КВАЗИИНВАРИАНТНЫЕ МЕРЫ НА НЕАРХИМЕДОВЫХ ПОЛУГРУППАХ ПЕТЕЛЬ}

\section{С.В. Людковский}

Далее $\mathbb{K}$ обозначает конечное алгебраическое расширение поля $p$-адических чисел $\mathbb{Q} p$. Пусть $\bar{M}$ и $N$ обозначают сепарабельные метризуемые многообразия, смоделированные на неархимедовых банаховых пространствах (БП) $X$ и $Y$ над полем $\mathbb{K}$. Предположим, что они имеют дизъюнктные аналитические атласы вида $\operatorname{At}(\bar{M})=\left\{\left(U_{j}, \phi_{j}\right): j \in \Upsilon\right\}$ и $\operatorname{At}(N)=$ $\left\{\left(P_{j}, \psi_{j}\right): j \in \Lambda\right\}$ и существуют вложения $\bar{M}$ в $X$ и $N$ в $Y$ в качестве открыто-замкнутых (ОЗ) подмножеств, где $U_{j}, C_{j}, P_{j}$ и $V_{j}$ ОЗ в $\bar{M}, X, N$ и $Y$ соответственно, $\phi_{j}: U_{j} \rightarrow C_{j}, \psi_{j}: P_{j} \rightarrow V_{j}$ являются гомеоморфизмами, задающими карты, $C_{j}$ ограничены в $X, \Lambda \cup \Upsilon \subset \mathbb{N}$. БП $X$ (и $Y$ ) изоморфно пространству $c_{0}(\alpha, \mathbb{K})\left(\right.$ и $\left.c_{0}(\beta, \mathbb{K})\right)$ сходящихся к нулю последовательностей $x=\left(x^{j} \in \mathbb{K}: j \in \alpha\right),\|x\|:=\max _{j}\left|x^{j}\right|_{\mathbb{K}}$ при $\alpha=\mathbb{N}$ и $X=\mathbb{K}^{m}$ при $\alpha=m \in \mathbb{N}$, где $\alpha$ и $\beta \geqslant 1-$ ординалы. Пусть $b \in \mathbb{Q}$ и $0<b<1$, тогда $j(b, z):=p^{b \times \operatorname{ord}_{p}(z)} \in \mathbb{C}_{p}$ $\forall z \in \mathbb{K}$, где $\mathbb{C}_{p}$ - поле комплексных чисел с неархимедовым нормированием, такое, что $\mathbb{C}_{p} \supset K, p^{-\operatorname{ord}_{p}(z)}:=|z|_{\mathbb{K}}$. Тогда, как и в [3], [4], через $C_{0}^{\theta}(t, s ; \bar{M} \rightarrow N)$ обозначим равномерное пространство отображений $f: \bar{M} \rightarrow N \mathrm{c} \bar{f}_{l, j}:=f_{l, j}-\theta_{l, j}, f_{l, j}:=\psi_{l} \circ f \circ \phi_{j}{ }^{-1}$ на $\phi_{j}\left(f^{-1}\left(P_{l}\right) \cap U_{j}\right) \neq \varnothing$ по базису $q_{i} \bar{Q}_{m}\left(x_{n}\right)$ с коэффициентами $a\left(m, \bar{f}^{i}\right) \in \mathbb{K}, \bar{f}_{l, j}=$ $\sum_{\left(n, i \in \mathbb{N}, m \in \mathbb{N}_{0}^{n}\right)} a\left(m, \bar{f}_{l, j}^{i}\right) \bar{Q}_{m}\left(x_{n}\right) q_{i}, \lim _{i+|m|+\operatorname{Ord}(m) \rightarrow \infty}\left|a\left(m, \bar{f}_{l, j}^{i}\right)\right| J(t, s, m)=0$, где для пространств $C$ без индекса 0 последнее условие не накладывается, $\left\{e_{j}: j \in \Upsilon\right\}$ и $\left\{q_{i}: i \in \Lambda\right\}-$ стандартные ортонормированные в неархимедовом смысле базисы (ОНБ) в $X$ и $Y, \bar{Q}_{m}\left(x_{n}\right)$ - базис полиномов Амиса на ОЗ подмножестве $V_{j, n}:=V_{j} \cap \mathbb{K}^{n}$ в $B\left(\mathbb{K}^{n}, z, r\right):=\left\{x \in \mathbb{K}^{n}:\|x-z\| \leqslant r\right\}, z \in \mathbb{K}^{n}, r>0, x_{n}:=\left(x^{1}, \ldots, x^{n}\right)$, Ord $(m):=$ $\max _{m_{i} \neq 0} i=n \in \mathbb{N}_{0}, Q_{0}(x):=1, m=\left\{m_{i}: i \in \Upsilon\right\}$, дробная часть числа $\{t\}=b, 0 \leqslant t \in \mathbb{Q}$, $s \in \mathbb{N}_{0}:=\mathbb{N} \cup\{0\}, \theta: \bar{M} \rightarrow N$ - фиксированное бесконечно дифференцируемое отображение. В частности, $C_{0}^{\theta}(t, s ; B(X, 0, r) \rightarrow Y)$ является БП с $\|f\|_{t, s}=\max _{i, n, m}\left|a\left(m, f^{i}\right)\right| J(t, s, m)$, где $J(t, s, m):=\sup _{\left(x \in V_{j, n}^{d}, v=v^{\prime}+(1, \ldots, 1) s^{\prime},\left|v^{\prime}\right| \leqslant t, 0 \leqslant s^{\prime} \leqslant s\right)}\left|\left(\Phi^{v} \bar{Q}_{m}\right)(x)\right|, \Phi^{v}$ - разделенные разности, $|v|=v^{1}+\cdots+v^{n}, \theta(B(X, 0, r))=\{0\}, d=[|v|]+1+\operatorname{sign}\{v\},[c]-$ целая часть числа $c \in \mathbb{R}$. Тогда при $0<b<1, b \in \mathbb{R},\{t\}=b$ определим $C_{0}^{\theta}(t, s ; B(X, 0, r) \rightarrow Y)$ как пополнение пространства бесконечно дифференцируемых функций по норме $\|f\|_{t, s}:=$ $\sup _{0 \leqslant w \leqslant t, w \in \mathbb{Q}}\|f\|_{w, s}$, для $\operatorname{dim}_{\mathbb{K}} M=\infty$ пусть $s=0$. Равномерность на $C_{0}^{\theta}(t, s ; \bar{M} \rightarrow N)$ задается с помощью строгого индуктивного предела пространств $C_{0}^{\theta}\left(t, s ; U^{j} \rightarrow N\right)=H_{j}$ с вложениями $\Psi_{l}^{j}: H_{j} \hookrightarrow H_{l} \forall j \leqslant l \in \Upsilon$, где $U^{j}=\bigcup_{i=1}^{j} U_{i}$.

Пусть $s_{0}=0 \in \bar{M}$ и $y_{0}=0 \in N, G(t, s):=\left\{g \in C^{\theta}(t, s ; \bar{M} \rightarrow \bar{M})\right\} \cap \operatorname{Hom}(\bar{M})$, где $\theta(x)=\operatorname{id}(x):=x \forall x \in M:=\bar{M} \backslash\left\{s_{0}\right\}, 0 \leqslant t \in \mathbb{Q}$. В пространстве $C_{0}^{0}\left(t, s ;\left(M, s_{0}\right) \rightarrow\right.$ $\left.\left(N, y_{0}\right)\right):=\left\{f \in C_{0}^{0}(t, s ; \bar{M} \rightarrow N) \mid\left(\bar{\Phi}^{j} f\right)\left(s_{0}\right)=0\right.$ при $\left|j^{\prime}\right|=0,1, \ldots, t ; j=j^{\prime}+s^{\prime}(1, \ldots, 1) ; 0 \leqslant$ $\left.s^{\prime} \leqslant s\right\}$ рассмотрим отношение эквивалентности $f K_{t, s} g$, которое означает, что $\exists \eta_{n} \in G(t, s)$, $\eta_{n}\left(s_{0}\right)=s_{0}, f_{n}$ и $g_{n} \in C_{0}^{0}\left(t, s ;\left(M, s_{0}\right) \rightarrow\left(N, y_{0}\right)\right)$, такие, что $f_{n}\left(\eta_{n}(x)\right)=g_{n}(x)$ для всех $x \in M, \lim _{n \rightarrow \infty} f_{n}=f, \lim _{n \rightarrow \infty} g_{n}=g$. В $C_{0}^{0}\left(t, s ;\left(M, s_{0}\right) \rightarrow\left(N, y_{0}\right)\right)$ введем композицию $f \circ g=: h$ с $h(x)=g\left(x_{1}\right)$ при $x \in M_{1}$ и $h(x)=f\left(x_{2}\right)$ при $x \in M_{2}$, где $x=\left(x_{1}, x_{2}\right) \in M_{1} \cup$ $M_{2} \subset M \times M, M_{1}:=M \times\left\{s_{0}\right\}, M_{2}=\left\{s_{0}\right\} \times M, \chi: M_{1} \cup M_{2} \rightarrow M$ - соответствующий $C(\infty)$-диффеоморфизм, $h(\chi(x)) \in C_{0}^{0}\left(t, s ;\left(M, s_{0}\right) \rightarrow\left(N, y_{0}\right)\right)$.

ОПРЕДЕЛЕниЕ 1. Факторпространство $C_{0}^{c^{0}}\left(t, s ;\left(M, s_{0}\right) \rightarrow\left(N, y_{0}\right)\right) / K_{t, s}$ назовем $n p o c m$ ранством петель и обозначим $\Omega_{t, s}(M, N)$, где $c^{0}(M)=\left\{y_{0}\right\}$.

Лемма 1. Пространство $\Omega_{t, s}(M, N)$ является хаусдорфовым, сепарабельнылм, ультраравномерным и полным со структурой топологического абелевого моноида (по- 
лугруппь с единичным әлементом); $\Omega_{t, s}(M, N)$ плотно в себе и имеет мощность $\mathrm{c}=\operatorname{card}(\mathbb{R})$.

Лемма 2. Для любьх $1 \leqslant t \in \mathbb{Q} u s \in \mathbb{N}_{0}$ пространство $\Omega_{t, s}(M, N)=: G$ является аналитическим многообразием и определено отображение $\bar{E}: \bar{V} \eta \rightarrow W_{\eta}$, являющееся $C^{\infty}$-диффеоморфизмом, $\bar{E}(V)=\overline{\exp }_{\eta(x)} \circ V_{\eta}$, где $\bar{V}_{\eta}-$ ОЗ окрестность нулевого сечения касательного расслоения $T_{\eta} G \subset T G, V_{\eta} \in \bar{V} \eta, G \supset W_{\eta} \ni \eta, \overline{\exp }: \widetilde{T} N \rightarrow N, \widetilde{T} N-$ окрестность $N$ в $T N, \overline{\exp }_{y}(z):=z$ для любьх $z \in B(Y, y, r) \subset T y N$ для соответствующux $0<r<\infty u y \in N \subset Y, T N=\bigcup_{y \in N} T y N \approx N \times Y$.

Доказательство вытекает из [4 (180), §3.4].

Теорема. Полугруппа $\Omega_{t, s}(M, N)=: G$ (см. лемму 2) имеет квазиинвариантную меру (КИМ) со значениями в $\mathbb{R} u \mathbb{Q}_{j}$ для простых $j$ с $j \neq p$.

Наметим путь доказательства. При $t \geqslant 1$ пространство $A^{k} G$ дифференциальных $k$-форм многообразия $G$ изоморфно $\Omega_{t, s}\left(M, A^{k} N\right) \times A_{y_{0}}^{k} N$, где $\theta(M)=\left\{y_{0} \times 0\right\}$ (см. леммы 1,2$)$, $k \in \mathbb{N}, k \leqslant \alpha$. Для $f \in C_{0}^{c^{0}}(t, s ; M \rightarrow N)$ и $\omega \in \Omega_{t, s}\left(M, A^{k} N\right)$ определено ограничение $f^{*} \omega \in C_{0}^{\theta}\left(t-1, s ; M \rightarrow A^{k} M\right)$ [1]. Для ограниченного $M$ в $X$, например, $U^{j}$ в качестве $M$, антидиффференцирование $P_{a}(l)$ на $C_{0}^{\theta}\left(t-1, s ; M_{a} \rightarrow Y\right)$ [3] индуцирует с учетом отношения эквивалентности $K_{t, s}$ непрерывньй аддитивный оператор $\left(\bar{P}_{a}(l) \bar{f}\right)^{*} \omega$ из $G_{a} \times \Omega_{t, s}\left(M, A^{k} N\right)$ в $\Omega_{t-1, s+1}\left(M_{a}, A^{k} M\right) \times A_{s_{0}}^{k} M \subset \Omega_{t, s}\left(M_{a}, A^{k} M\right) \times A_{s_{0}}^{k} M$, где $f \in \bar{f} \in G_{a}, M_{a}=M \cap \mathbb{K}^{a}$, $G_{a}:=\Omega_{t, s}\left(M_{a}, N\right) \hookrightarrow G, a \in \mathbb{N}, l=[t-1], s_{0} \in M_{a}$. Это отображение инъективно при $\alpha \geqslant \beta, H_{a}:=A_{c^{0}}^{k} \Omega_{t-1, s+1}\left(M_{a}, M\right)$. При $\alpha<\beta=: \gamma$ рассмотрим пространство $Z:=c_{0}\left(\left\{H_{a, n}: a \in \mathbb{N}^{c^{0}} n \in \gamma\right\}\right):=\left\{z=\left(z^{a, n} \in H_{a, n}\right):\|z\|=\sup _{a, n}\left\|z^{a, n}\right\|_{H_{a, n}} ;\right.$ $\left.\lim _{a, n}\left\|z^{a, n}\right\|_{H_{a, n}}=0\right\}$ при $\gamma=\mathbb{N}$, пространство $Z:=\bigotimes_{a, n} H_{a, n}$ при $\alpha$ и $\gamma \in \mathbb{N}$, а при $\alpha>\beta$ пусть $Z:=c_{0}\left(H_{a}: a\right)$, где $H_{a}=H_{a, n}$. Существуют $w_{i, j} \in \Omega_{\infty}\left(M, A^{k} N\right)$, $\xi_{a, n} \in \mathbb{K}$ и $h_{a, n} \in H_{a, n}$, такие, что $A(v):=\sum_{a, i, n} \xi_{a, n}\left(\bar{P}_{a}(l)\left(\bar{f} \mid M_{a}\right)\right)^{*} w_{i, n} h_{a, n}-$ инъективньй аддитивный оператор из $\bar{V}_{\zeta}$ в $Z,\|A\|$ и $\left\|A^{-1}\right\| \in[1 / p, 1]$, где $\bar{E}(v)=f$ (см. леммы 1,2$)$. Пусть $G^{\prime}=C_{0,\{k\}}^{c^{0}}(t, s ; M \rightarrow N) / K_{t, s}$ - плотная подполугруппа в $G$ с $C_{0,\{k\}}^{0}(t, s ; M \rightarrow Y):=\left\{\phi \in C_{0}^{0}(t, s ; M \rightarrow Y)\left|\lim _{k(i, m) \rightarrow \infty}\right| a\left(m, \phi^{i}\right) \mid J(t, s ; m) p^{k(i, m)}=0 ;\right.$ $\left.\|\phi\|=\sup _{i, m}\left|a\left(m, \phi^{i}\right)\right| J(t, s ; m) p^{k(i, m)}\right\}$, где $k(i, m)=c \times(i+|m|+\operatorname{Ord}(m)), c=$ const $>1$. Тогда КИМ на банаховом подпространстве $s p_{\mathbb{K}} A(\bar{V})$ в $Z$ с помощью оператора $A$ индуцирует КИМ сначала на $G$ для $U^{j}$, а с помощњю индуктивного предела на $G$ для $M=\bigcup_{j} U^{j}$, в силу [4 (210), теоремы 3.23 и 3.28$]$.

\section{СПИСОК ЛИТЕРАТУРЫ}

[1] Бурбаки Н. Многообразия. М.: Мир, 1975. [2] Энгелькинг Р. Общая топология. М.: Мир, 1986. [3] Людковский С. В. // УМН. 1996. Т. 51. №2. С. 169-170. [4] Людковский C. B. Preprints IC/96/180, 181, 210, 215. Trieste: ICTP, 1996. [5] Van Rooij A. C. M. NonArchimedean functional analysis. New York: Marcel Dekker, 1978. 